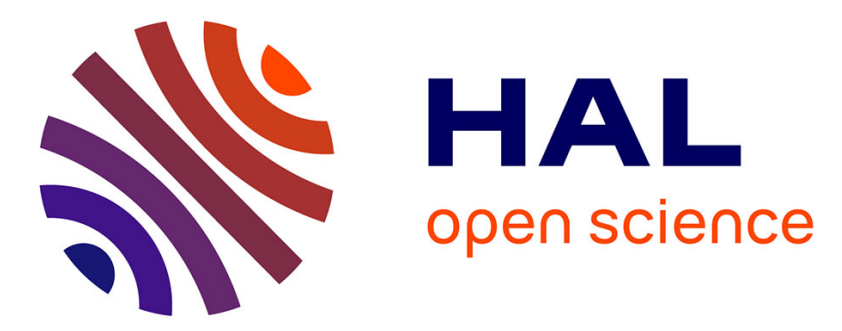

\title{
Discussion on: "Sensor gain fault diagnosis for a class of nonlinear systems"
}

Hugues Rafaralahy, Michel Zasadzinski, Mohamed Boutayeb

\section{To cite this version:}

Hugues Rafaralahy, Michel Zasadzinski, Mohamed Boutayeb. Discussion on: "Sensor gain fault diagnosis for a class of nonlinear systems". European Journal of Control, 2006, 12 (5), pp.536-544. hal-00143450

\section{HAL Id: hal-00143450 \\ https://hal.science/hal-00143450}

Submitted on 25 Apr 2007

HAL is a multi-disciplinary open access archive for the deposit and dissemination of scientific research documents, whether they are published or not. The documents may come from teaching and research institutions in France or abroad, or from public or private research centers.
L'archive ouverte pluridisciplinaire HAL, est destinée au dépôt et à la diffusion de documents scientifiques de niveau recherche, publiés ou non, émanant des établissements d'enseignement et de recherche français ou étrangers, des laboratoires publics ou privés. 


\title{
Discussion on: "Sensor Gain Fault Diagnosis for a Class of Nonlinear Systems"
}

\author{
H. Rafaralahy ${ }^{\dagger *}$, M. Zasadzinski ${ }^{\dagger}$ and M. Boutayeb ${ }^{\ddagger}$ \\ $\dagger$ CRAN - CNRS, IUT de Longwy, Université Henri Poincaré - Nancy Université \\ 186 rue de Lorraine, 54400 Cosnes et Romain, France \\ $\ddagger$ LSIIT -CNRS, Université Louis Pasteur - Strasbourg I \\ Pôle API, Boulevard Sébastien Brant, 67400 Illkirch, France \\ E-mails : Hugues.Rafaralahy@iut-longwy.uhp-nancy.fr, Michel.Zasadzinski@iut-longwy.uhp-nancy.fr, \\ Mohamed.Boutayeb@ipst-ulp.u-strasbg.fr
}

\section{Introduction}

The paper by Y. Wang and D. H. Zhou [1] presents an approach of sensor gain fault detection of nonlinear systems. The fisrt step of this approach consists on the location of the sensor gain faults that can be estimated. The proposed algorithm is based on the computation of the failure matrix $\mathrm{D}$ such that the zeros of the linear part of the system $(A, 0, C, D)$ are stable. The second step is the simultaneous estimation of the state and the fault vectors using the singular systems representation given in [2]. Finally, the sensor gain faults are estimated using an adaptive estimator. This paper is an interesting contribution to the sensor gain faults diagnosis of nonlinear systems provided that the proposed approach gives both the location and the estimation of the failure. This discussion focuses on the existence conditions of the simultaneous estimation of the state and the fault vector and on the selection of the conditionnally identifiable faults. It is shown first that the detectability of the pair $(A, C)$ is not necessary and only the measurement provided by the conditionally detectable sensors are necessary to estimate the state of the system. An estimation of the fault vector $f$ is then given by the output of the observer using the conditionally identifiable sensors. Thereafter, we claim that the simultaneous estimation scheme can be simplified using a low order observer and without using descriptor systems formulation. An $n^{\text {th }}$-order state observer and a $q^{\text {th }}$-order functional observer which estimates the conditionally identifiable faults are then proposed. Finally, an algorithm to determine the matrix $D$ based on a detectability condition is exhibited.

\section{On the existence conditions of the observer}

This section is devoted to the analysis of the existence conditions of the observer proposed in [1] when the singular systems description is used. More precisely, the necessary condition of the linear part of the system is investigated using an output transformation. The singular state-space formulation is therefore not necessary.

\subsection{Problem reformulation using an output transformation}

In this section, it is assumed that the conditionnally identifiable sensor gain faults, i.e. the matrix $D$, have been computed using the algorithm proposed by Y. Wang and D. H. Zhou [1]. The considered system is given by eq. (12) in the paper [1]

$$
\dot{x}=A x+g(x, u, t)+B u
$$

\footnotetext{
${ }^{*}$ Corresponding author : E-mail: Hugues.Rafaralahy@iut-longwy.uhp-nancy.fr - Fax : +33 +3 82396291
} 


$$
y=C x+D f_{D}
$$

with $x \in \mathbb{R}^{n}, f_{D} \in \mathbb{R}^{q}, y \in \mathbb{R}^{m}$ and $\operatorname{rank} D=q$. As in [1] (Assumption 2), $g(x, u, t)$ is assumed to be globally Lipschitz with respect to $x$ :

$$
\left\|g\left(x_{1}, u, t\right)-g\left(x_{2}, u, t\right)\right\| \leqslant \lambda\left\|x_{1}-x_{2}\right\|, \forall u, \forall t \geqslant 0
$$

where $\lambda>0$ is the Lipschitz constant.

First, we propose an output transformation to isolate the free fault component $y_{1}$ of the measurement equation to the faulty measurement $y_{2}$. Thereafter, it will be shown that only $y_{1}$ is necessary to estimate the state $x$ and $y_{2}$ is only used to deduce the sensor fault estimation $f$ using the state estimation. Recall that the matrix $D$ is of full column rank, i.e. $\operatorname{rank} D=q$, with at most only one ' 1 ' in each row. Then there exist two non singular permutation matrices $V \in \mathbb{R}^{m \times m}$ and $W \in \mathbb{R}^{q \times q}$ such that

$$
V D W=\left[\begin{array}{c}
0 \\
I_{q}
\end{array}\right], V C=\left[\begin{array}{l}
C_{1} \\
C_{2}
\end{array}\right],\left[\begin{array}{l}
y_{1} \\
y_{2}
\end{array}\right]=V y \text { and } f=W^{T} f_{D}
$$

Using this output transformation, the system (1) is then equivalent to

$$
\begin{aligned}
\dot{x} & =A x+g(x, u, t)+B u \\
y_{1} & =C_{1} x \\
y_{2} & =C_{2} x+f
\end{aligned}
$$

with $y_{1} \in \mathbb{R}^{m_{1}}\left(m=m_{1}+q\right)$. In the sequel and without loss of generality, we assume that the system is of the form (4). Notice that equations (4b) and (4c) correspond to the structure of matrix $D$ given by eq. (7) in $[1]$.

\subsection{State and sensor fault estimation using a singular systems framework}

\subsubsection{Existence conditions of the observer}

Using the approach given in [2], the authors rewrite system (4) as a singular system as follows

$$
\begin{aligned}
E \dot{\bar{x}} & =M \bar{x}+g(x, u, t)+B u \\
y & =H \bar{x}
\end{aligned}
$$

with

$$
\bar{x}=\left[\begin{array}{l}
x \\
f
\end{array}\right], E=\left[\begin{array}{ll}
I_{n} & 0
\end{array}\right], M=\left[\begin{array}{ll}
A & 0
\end{array}\right], H=\left[\begin{array}{cc}
C_{1} & 0 \\
C_{2} & I_{q}
\end{array}\right]
$$

It is easy to see that system (5) is observable at infinity [3] since $\operatorname{rank}\left[\begin{array}{c}E \\ H\end{array}\right]=\operatorname{dim} \bar{x}=n+q$. It follows from this property that the necessary condition for the existence of an observer is reduced to the following detectability condition $[3,4]$

$$
\operatorname{rank}\left[\begin{array}{c}
\mu E-M \\
H
\end{array}\right]=n+q, \forall \mu \in \mathbb{C}, \operatorname{Re}(\mu) \geqslant 0
$$

which can be expressed (see [2]) as minimum phase condition by the use of the expressions of matrices $E, M$ and $H$

$$
\begin{aligned}
& \operatorname{rank}\left[\begin{array}{c}
\mu E-M \\
H
\end{array}\right]=\operatorname{rank}\left[\begin{array}{cc}
\mu I_{n}-A & 0 \\
C & D
\end{array}\right]=n+q, \forall \mu \in \mathbb{C}, \operatorname{Re}(\mu) \geqslant 0 \\
\Longleftrightarrow & \operatorname{rank}\left[\begin{array}{cc}
\mu I_{n}-A & 0 \\
C_{1} & 0 \\
C_{2} & I_{q}
\end{array}\right]=n+q, \forall \mu \in \mathbb{C}, \operatorname{Re}(\mu) \geqslant 0 \\
\Longleftrightarrow & \operatorname{rank}\left[\begin{array}{c}
\mu I_{n}-A \\
C_{1}
\end{array}\right]=n, \forall \mu \in \mathbb{C}, \operatorname{Re}(\mu) \geqslant 0
\end{aligned}
$$


The detectability of the singular system (5) is then reduced to the detectability of the pair $\left(A, C_{1}\right)$ which is more tractable than condition (13) in [1]. Thus, the detectability of the pair $(A, C)$ given by Assumption 1 in [1] is not necessary. Note first that the detectability of the pair $(A, C)$ implies the detectability of the pair $\left(A, C_{1}\right)$ and the converse is not true. For linear systems, i.e. $g(x, u, t)=0$, the detectability of the pair $\left(A, C_{1}\right)$ is a necessary and sufficient condition for observer design. In addition, as it will be shown in section 4 , detectability condition (8) is more tractable for the computation of the matrix $D$.

\subsubsection{On the relations between the choice of the generalized inverse and the observer's structure}

This section is devoted to the choice of the generalized inverse of the matrix $\left[\begin{array}{c}E \\ H\end{array}\right]$. It will be shown that the system description (5) makes it possible to light the parts of $y_{1}$ and $y_{2}$ in the observer design. The proposed observer given in [1] (eq. (20)) can be rewritten as follows

$$
\begin{aligned}
\dot{z} & =N z+L_{1} y_{1}+L_{2} y_{2}+P g(\widehat{x}, u, t)+P B u \\
\widehat{x} & =z_{1}+Q_{11} y_{1}+Q_{12} y_{2} \\
\widehat{f} & =z_{2}+Q_{21} y_{1}+Q_{22} y_{2}
\end{aligned}
$$

with $z_{1} \in \mathbb{R}^{n}, z_{2} \in \mathbb{R}^{q}$ and

$$
z=\left[\begin{array}{l}
z_{1} \\
z_{2}
\end{array}\right], P=\left[\begin{array}{l}
P_{1} \\
P_{2}
\end{array}\right], Q=\left[\begin{array}{ll}
Q_{11} & Q_{12} \\
Q_{21} & Q_{22}
\end{array}\right]
$$

where matrices $P$ and $Q$ are given by the generalized inverse of the full rank matrix $\left[\begin{array}{l}E \\ H\end{array}\right]$

$$
\left[\begin{array}{ll}
P & Q
\end{array}\right]=\left[\begin{array}{l}
E \\
H
\end{array}\right]^{\dagger} \quad \text { such that } \quad\left[\begin{array}{l}
E \\
H
\end{array}\right]^{\dagger}\left[\begin{array}{l}
E \\
H
\end{array}\right]=I_{n+q}
$$

All generalized inverses of full rank matrices can be parametrized by matrices $\alpha$ with $\operatorname{det} \alpha \neq 0$ and $\beta$ such that $[5]$

$$
\alpha\left[\begin{array}{c}
E \\
H
\end{array}\right]=\left[\begin{array}{l}
E_{1} \\
E_{2}
\end{array}\right] \text { with } \operatorname{det} E_{1} \neq 0 \text { and }\left[\begin{array}{c}
E \\
H
\end{array}\right]^{\dagger}=\left[\left(E_{1}^{-1}-\beta E_{2} E_{1}^{-1}\right) \quad \beta\right] \alpha
$$

Applying relation (11) with

$$
\alpha=\left[\begin{array}{ccc}
I_{n} & 0 & 0 \\
0 & 0 & I_{q} \\
0 & I_{m_{1}} & 0
\end{array}\right],\left[\begin{array}{l}
E_{1} \\
E_{2}
\end{array}\right]=\left[\begin{array}{cc}
I_{n} & 0 \\
C_{2} & I_{q} \\
\hline C_{1} & 0
\end{array}\right], E_{1}^{-1}=\left[\begin{array}{cc}
I_{n} & 0 \\
-C_{2} & I_{q}
\end{array}\right] \text { and } \beta=\left[\begin{array}{l}
\beta_{1} \\
\beta_{2}
\end{array}\right]
$$

yields

$$
\left[\begin{array}{l}
E \\
H
\end{array}\right]^{\dagger}=\left[\begin{array}{ccc}
I_{n}-\beta_{1} C_{1} & \beta_{1} & 0 \\
-C_{2}-\beta_{2} C_{1} & \beta_{2} & I_{q}
\end{array}\right]=\left[\begin{array}{lll}
P_{1} & Q_{11} & Q_{12} \\
P_{2} & Q_{21} & Q_{22}
\end{array}\right]
$$

Then, for all generalized inverses of $\left[\begin{array}{c}E \\ H\end{array}\right]$, we have $Q_{12}=0$ and $Q_{22}=I_{q}$. In addition, matrices $Q_{11}$ and $Q_{21}$ are arbitrary since $Q_{11}=\beta_{1}$ and $Q_{21}=\beta_{2}$.

Using relations (24) and (25) in [1] and the generalized inverse (13), one obtains

$$
\begin{aligned}
{\left[\begin{array}{ll}
L_{1} & L_{2}
\end{array}\right]=F+N Q=F+} & (P M-F H) Q=F(I-H Q)+P M Q \\
& =\left[\begin{array}{ll}
F_{11} & F_{12} \\
F_{21} & F_{22}
\end{array}\right]\left[\begin{array}{cc}
I_{n}-C_{1} \beta_{1} & 0 \\
-C_{2} \beta_{1}-\beta_{2} & 0
\end{array}\right]+\left[\begin{array}{cc}
\left(I_{n}-\beta_{1} C_{1}\right) A \beta_{1} & 0 \\
-\left(C_{2}+\beta_{2} C_{1}\right) A \beta_{1} & 0
\end{array}\right]=\left[\begin{array}{ll}
L_{1} & 0
\end{array}\right]
\end{aligned}
$$

or equivalently $L_{2}=0$ for all generalized inverses of matrix $\left[\begin{array}{c}E \\ H\end{array}\right]$, and the observer (9) becomes

$$
\dot{z}=N z+L_{1} y_{1}+P g(\widehat{x}, u, t)+P B u
$$




$$
\begin{aligned}
& \widehat{x}=z_{1}+\beta_{1} y_{1} \\
& \widehat{f}=z_{2}+\beta_{2} y_{1}+y_{2}
\end{aligned}
$$

Notice that, for all generalized inverses of $\left[\begin{array}{c}E \\ H\end{array}\right]$, only measurement $y_{1}$ is needed in the state observer (15a) and for the estimation $\widehat{x}$ in (15b) while the measurement $y_{2}$ is used to deduce the fault estimation.

If the Moore-Penrose generalized inverse is used as in [1] (see eq. (19) in [1]), one obtains

$$
\begin{aligned}
& {\left[\begin{array}{ll}
P & Q
\end{array}\right]=\left[\begin{array}{l}
E \\
H
\end{array}\right]^{\dagger}=\left(\left[\begin{array}{ll}
E^{T} & H^{T}
\end{array}\right]\left[\begin{array}{l}
E \\
H
\end{array}\right]\right)^{-1}\left[\begin{array}{ll}
E^{T} & H^{T}
\end{array}\right]} \\
& =\left[\begin{array}{ccc}
\left(I_{n}+C_{1}^{T} C_{1}\right)^{-1} & \left(I_{n}+C_{1}^{T} C_{1}\right)^{-1} C_{1}^{T} & 0 \\
-C_{2}\left(I_{n}+C_{1}^{T} C_{1}\right)^{-1} & -C_{2}\left(I_{n}+C_{1}^{T} C_{1}\right)^{-1} C_{1}^{T} & I_{q}
\end{array}\right]=\left[\begin{array}{ccc}
P_{1} & Q_{11} & Q_{12} \\
P_{2} & Q_{21} & Q_{22}
\end{array}\right]
\end{aligned}
$$

which corresponds to

$$
\beta=\left[\begin{array}{c}
\beta_{1} \\
\beta_{2}
\end{array}\right]=\left[\begin{array}{c}
\beta_{1} \\
-C_{2} \beta_{1}
\end{array}\right]=\left[\begin{array}{c}
\left(I_{n}+C_{1}^{T} C_{1}\right)^{-1} C_{1}^{T} \\
-C_{2}\left(I_{n}+C_{1}^{T} C_{1}\right)^{-1} C_{1}^{T}
\end{array}\right]=\left[\begin{array}{c}
Q_{11} \\
Q_{21}
\end{array}\right]
$$

A more simple generalized inverse can be choosen as follows

$$
\left[\begin{array}{ll}
\bar{P} & \bar{Q}
\end{array}\right]=\left[\begin{array}{c}
E \\
H
\end{array}\right]^{\dagger}=\left[\begin{array}{lll}
\bar{P}_{1} & \bar{Q}_{11} & \bar{Q}_{12} \\
\bar{P}_{2} & \bar{Q}_{21} & \bar{Q}_{22}
\end{array}\right]=\left[\begin{array}{ccc}
I_{n} & 0 & 0 \\
-C_{2} & 0 & I_{q}
\end{array}\right]
$$

which corresponds to the case $\beta=0$ and the observer (9) or (15) can be simplified as

$$
\begin{aligned}
\dot{z} & =N z+L_{1} y_{1}+L_{2} y_{2}+\bar{P} g(\widehat{x}, u, t)+\bar{P} B u \\
\widehat{x} & =z_{1} \\
\widehat{f} & =z_{2}+y_{2}
\end{aligned}
$$

In comparison with the observer (15), the measurement $y_{1}$ does not act in an explicit way on the outputs $\widehat{x}$ and $\widehat{f}$ of the observer. by

Using again relations (24) and (25) in [1] and the generalized inverse (18), the observer (19) is given

$$
N=\bar{P} M-F H=\left[\begin{array}{c}
I_{n} \\
-C_{2}
\end{array}\right]\left[\begin{array}{ll}
A & 0
\end{array}\right]-\left[\begin{array}{ll}
F_{11} & F_{12} \\
F_{21} & F_{22}
\end{array}\right]\left[\begin{array}{ll}
C_{1} & 0 \\
C_{2} & I_{q}
\end{array}\right]=\left[\begin{array}{cc}
A-F_{11} C_{1}-F_{12} C_{2} & -F_{12} \\
-C_{2} A-F_{21} C_{1}-F_{22} C_{2} & -F_{22}
\end{array}\right]
$$

and

$$
\left[\begin{array}{ll}
L_{1} & L_{2}
\end{array}\right]=F+N \bar{Q}=\left[\begin{array}{ll}
F_{11} & F_{12} \\
F_{21} & F_{22}
\end{array}\right]+\left[\begin{array}{cc}
A-F_{11} C_{1}-F_{12} C_{2} & -F_{12} \\
-C_{2} A-F_{21} C_{1}-F_{22} C_{2} & -F_{22}
\end{array}\right]\left[\begin{array}{ll}
0 & 0 \\
0 & I_{q}
\end{array}\right]=\left[\begin{array}{ll}
F_{11} & 0 \\
F_{21} & 0
\end{array}\right]
$$

Remark 1. For all generalized inverses of matrix $\left[\begin{array}{c}E \\ H\end{array}\right]$ we have $Q_{12}=0$ and $L_{2}=0$ then $y_{2}$ is not used in the dynamics of the observer nor in the state estimation $\widehat{x} . y_{2}$ acts only on the fault estimation $\widehat{f}$ (observers output) what suggests using only relations (4a) and (4b) to estimate $x$. In addition, for the choice of the generalized inverse given by (18), the measurement $y_{1}$ affects the estimations of $x$ and $f$ after being filtered in (19a), i.e. the measurement noises in $y_{1}$ are filtered in (19). This is not the case if the Moore-Penrose generalized inverse is chosen as in [1].

Remark 2 (The Moore-Penrose generalized inverse with block-diagonal measurement output matrix). Without loss of generality, assume that $\operatorname{rank} C=m$ or $\operatorname{rank} C_{1}=m_{1}$ and $\operatorname{rank} C_{2}=q$ with $m=m_{1}+q$. Then there exists a non singular matrix $G \in \mathbb{R}^{n \times n}$ such that

$$
\left[\begin{array}{cc}
\bar{C}_{1} & 0 \\
0 & \bar{C}_{2}
\end{array}\right]=\left[\begin{array}{l}
C_{1} \\
C_{2}
\end{array}\right] G, \widetilde{x}=\left[\begin{array}{c}
\widetilde{x}_{1} \\
\widetilde{x}_{2}
\end{array}\right]=G^{-1} x, \widetilde{A}=G^{-1} A G, \widetilde{g}(\widetilde{x}, u, t)=G^{-1} g(x, u, t), \widetilde{B}=G^{-1} B
$$


then the system (4) is equivalent to

$$
\begin{aligned}
\dot{\widetilde{x}} & =\widetilde{A} \widetilde{x}+\widetilde{g}(\widetilde{x}, u, t)+\widetilde{B} u \\
y_{1} & =\bar{C}_{1} \widetilde{x}_{1} \\
y_{2} & =\bar{C}_{2} \widetilde{x}_{2}+f
\end{aligned}
$$

with $\widetilde{x}_{1} \in \mathbb{R}^{n_{1}}, \widetilde{x}_{2} \in \mathbb{R}^{n_{2}}$ et $n=n_{1}+n_{2}$. The Moore-Penrose generalized inverse (16) gives

$$
\begin{aligned}
{\left[\begin{array}{ll}
\widetilde{P} & \widetilde{Q}
\end{array}\right] } & =\left[\begin{array}{l}
E \\
H
\end{array}\right]^{\dagger}=\left(\left[\begin{array}{ll}
E^{T} & H^{T}
\end{array}\right]\left[\begin{array}{c}
E \\
H
\end{array}\right]\right)^{-1}\left[\begin{array}{ll}
E^{T} & H^{T}
\end{array}\right] \\
& =\left[\begin{array}{cc|c|c}
\left(I_{n_{1}}+\bar{C}_{1}^{T} \bar{C}_{1}\right)^{-1} & 0 & \left(I_{n_{1}}+\bar{C}_{1}^{T} \bar{C}_{1}\right)^{-1} \bar{C}_{1}^{T} & 0 \\
0 & I_{n_{2}} & 0 & 0 \\
\hline 0 & -\bar{C}_{2} & 0 & I_{q}
\end{array}\right]=\left[\begin{array}{ccc}
\widetilde{P}_{1} & \widetilde{Q}_{11} & \widetilde{Q}_{12} \\
\widetilde{P}_{2} & \widetilde{Q}_{21} & \widetilde{Q}_{22}
\end{array}\right]
\end{aligned}
$$

and the observer (15) becomes

$$
\begin{aligned}
\dot{\widetilde{z}} & =\widetilde{N} \widetilde{z}+\widetilde{L}_{1} y_{1}+\widetilde{P} \widetilde{g}(\widehat{\widetilde{x}}, u, t)+\widetilde{P} \widetilde{B} u \\
\widehat{\widetilde{x}} & =\widetilde{z}_{1}+\widetilde{Q}_{11} y_{1} \\
\widehat{f} & =\widetilde{z}_{2}+y_{2}
\end{aligned}
$$

Using (13), the observation error $\bar{e}=\widehat{\bar{x}}-\bar{x}$ can be rewritten as

$$
\bar{e}=z+\left(Q H-I_{n+q}\right) \bar{x}=\left[\begin{array}{l}
z_{1} \\
z_{2}
\end{array}\right]+\left(\left[\begin{array}{cc}
Q_{11} & 0 \\
Q_{21} & I_{q}
\end{array}\right]\left[\begin{array}{cc}
C_{1} & 0 \\
C_{2} & I_{q}
\end{array}\right]-\left[\begin{array}{cc}
I_{n} & 0 \\
0 & I_{q}
\end{array}\right]\right)\left[\begin{array}{l}
x \\
f
\end{array}\right]=\left[\begin{array}{l}
z_{1}+\left(Q_{11} C_{1}-I_{n}\right) x \\
z_{2}+\left(Q_{21} C_{1}+C_{2}\right) x
\end{array}\right]
$$

For the Moore-Penrose generalized inverse (16) used in [1], we obtain

$$
\bar{e}=\left[\begin{array}{c}
z_{1}-\left(\left(I_{n}+C_{1}^{T} C_{1}\right)^{-1}\right) x \\
z_{2}+\left(C_{2}\left(I_{n}+C_{1}^{T} C_{1}\right)^{-1}\right) x
\end{array}\right]
$$

Then in the observer (15) with generalized inverse (16), $z_{1}$ is an estimation of $\left(I_{n}+C_{1}^{T} C_{1}\right)^{-1} x$ and $z_{2}$ is an estimation of $-C_{2}\left(I_{n}+C_{1}^{T} C_{1}\right)^{-1} x=-C_{2} z_{1}$. For the observer (19), the observation error becomes

$$
\bar{e}=\left[\begin{array}{c}
z_{1}-x \\
z_{2}+C_{2} x
\end{array}\right]
$$

and $z_{1}$ is then an estimation of $x$ while $z_{2}$ is an estimation of $-C_{2} x=-C_{2} z_{1}$. In both cases, $z_{2}$ is not useful since it is a linear combination of $z_{1}$. Thus, it seems interesting to estimate either $x\left(n^{\text {th }}\right.$-order observer) or $C_{2} x$ ( $q^{\text {th }}$-order observer) without using a singular system framework. That will be the topics of sections 3.1 and 3.2.

\subsubsection{On the LMI (eq. (27) in [1]) and sufficient conditions}

The parameter $\mu>0$ in the LMI (eq. (27) in [1]) is not useful since left and rigth-multiplying this LMI by $\frac{1}{\lambda \sqrt{\mu}} I_{2 n+q}$ yields

$$
\left[\begin{array}{cc}
\bar{X} P M+M^{T} P^{T} \bar{X}-R H-H^{T} R^{T}+I_{n+q} & \bar{X} P \\
P^{T} \bar{X} & \frac{-1}{\lambda^{2}} I_{n}
\end{array}\right]<0
$$

Then the observer proposed by Wang et Zhou [1] exists if [6]

1. the function $g(x, t, u)$ verifies the Lipschitz condition (2),

2. there exist matrices $R$ and $\bar{X}=\bar{X}^{T}>0$ such that the LMI (29) is satisfied with $F=R^{-1} \bar{X}$ and $N=P M-F H$.

The above conditions are equivalent to the existence of a gain matrix $F$ such that $(P M-F H)$ is stable and $\left\|\left(s I_{n+q}-(P M-F H)\right)^{-1} P\right\|_{\infty}<\frac{1}{\lambda}$ where

$$
\dot{\bar{e}}=(P M-F H) \bar{e}+P g(\widehat{x}, u, t)-g(x, u, t))
$$




\section{State and sensor fault estimation without the singular systems frame- work}

It was shown in the previous development that the measurement $y_{2}$ is not used in the dynamics of the observer nor in the state estimation $\widehat{x}$ and a necessary condition is the detectability of the pair $\left(A, C_{1}\right)$. The main idea is then the synthesis of an $n^{\text {th }}$ order observer which dynamics has only $y_{1}$ as input and $y_{2}$ is used in the observers output $\widehat{f}$.

\section{$3.1 n^{\text {th }}$ order observers synthesis}

By the use of the preceding arguments and as suggested by the structure of the observer (15) it is rather normal to propose an $n^{\text {th }}$ order Kalman like observer of the following form

$$
\begin{aligned}
\dot{\widehat{x}} & =A \widehat{x}+g(\widehat{x}, u, t)+B u+L\left(y_{1}-C_{1} \widehat{x}\right) \\
\widehat{f} & =y_{2}-C_{2} \widehat{x}
\end{aligned}
$$

It was proved by Rajamani [6] that the dynamical system (31a) is an observer for the system (4a)-(4b) if there exists a gain matrix $L$ verifying $\left(A-L C_{1}\right)$ stable (i.e. the pair $\left(A, C_{1}\right)$ must be detectable) and $\left\|\left(s I_{n}-\left(A-L C_{1}\right)\right)^{-1}\right\|_{\infty}<\frac{1}{\lambda}$ or equivalently if there exist matrices $X=X^{T}>0$ and $Z$ such that the following LMI is satisfied

$$
\left[\begin{array}{cc}
X A+A^{T} X-Z C_{1}-C_{1}^{T} Z^{T}+I_{n} & X \\
X & \frac{-1}{\lambda^{2}} I_{n}
\end{array}\right]<0
$$

with $L=X^{-1} Z$. Then we have

$$
\left.\dot{e}=\left(A-L C_{1}\right) e+g(\widehat{x}, u, t)-g(x, u, t)\right)
$$

The observation error $e=\widehat{x}-x$ is then asymptotically stable. Now, let $e_{f}=\widehat{f}-f$ be the fault estimation error then

$$
e_{f}=\left(C_{2} x+f-C_{2} \widehat{x}\right)-f=-C_{2} e
$$

thus one can see thaf $e_{f}$ decays to zero if $e$ is asymptotically stable. Notice that, under the existence conditions given above there exists a reduced-order observer for the Lipschitz nonlinear system (4) (see $[7])$.

\section{$3.2 q^{\text {th }}$ order functional observer}

An another approach consists on using a $q^{\text {th }}$ order functional observer which estimates $C_{2} x$ since the estimation of the state $x$ is not necessarily useful to identify the faults $f$. Using (4c), let $\nu$ be the functional to be estimated

$$
\nu=C_{2} x
$$

with $\nu \in \mathbb{R}^{q}$. Without loss of generality, we assume that

$$
\operatorname{rank} C=\operatorname{rank}\left[\begin{array}{l}
C_{1} \\
C_{2}
\end{array}\right]=\operatorname{rank} C_{1}+\operatorname{rank} C_{2}=m
$$

A $q^{\text {th }}$ order functional observer can be expressed as follows

$$
\begin{aligned}
\dot{w} & =N w+L y_{1}+P g(\widehat{\nu}, u, t)+P B u \\
\widehat{\nu} & =w+Q y_{1} \\
\widehat{f} & =y_{2}-\widehat{\nu}
\end{aligned}
$$

with $w \in \mathbb{R}^{q}$. 
Necessary conditions for the existence of the observer (37) are given by [8]

$$
\begin{aligned}
\operatorname{rank}\left[\begin{array}{c}
C_{2} A \\
C_{1} A \\
C_{1} \\
C_{2}
\end{array}\right] & =\operatorname{rank}\left[\begin{array}{c}
C_{1} A \\
C_{1} \\
C_{2}
\end{array}\right] \\
\operatorname{rank}\left[\begin{array}{c}
\mu C_{2}-C_{2} A \\
C_{1} A \\
C_{1}
\end{array}\right] & =\operatorname{rank}\left[\begin{array}{c}
C_{1} A \\
C_{1} \\
C_{2}
\end{array}\right], \forall \mu \in \mathbb{C}, \operatorname{Re}(\mu) \geqslant 0
\end{aligned}
$$

In the case of functional observers for nonlinear Lipschitz systems, the necessary condition (38b) replaces the detectability condition (8). The synthesis of functional observer (37) takes as a starting point the results in $[8,9,10]$. The matrices of the functional observer are given by

$$
\begin{aligned}
N & =\left(C_{2} A \mathcal{M}^{\dagger}\right) \mathcal{M}_{\mathcal{N}}-\mathcal{K}_{\mathcal{M}}\left(\mathcal{I}_{\Uparrow+\mathbb{I}_{\infty}}-\mathcal{M} \mathcal{M}^{\dagger}\right) \mathcal{M}_{\mathcal{N}}=\mathcal{N}_{\infty}-\mathcal{K}_{\mathcal{M}} \mathcal{N}_{\epsilon} \\
Q & =\left(C_{2} A \mathcal{M}^{\dagger}\right) \mathcal{M}_{\mathcal{Q}}-\mathcal{K}_{\mathcal{M}}\left(\mathcal{I}_{\mathbb{1}+\mathbb{I}_{\infty}}-\mathcal{M} \mathcal{M}^{\dagger}\right) \mathcal{M}_{\mathcal{Q}}=\mathcal{Q}_{\infty}-\mathcal{K}_{\mathcal{M}} \mathcal{Q}_{\epsilon} \\
L & =\left(C_{2} A \mathcal{M}^{\dagger}\right) \mathcal{M}_{\mathcal{L}}-\mathcal{K}_{\mathcal{M}}\left(\mathcal{I}_{\mathbb{1}+\mathbb{I}_{\infty}}-\mathcal{M} \mathcal{M}^{\dagger}\right) \mathcal{M}_{\mathcal{L}}+\mathcal{N} \mathcal{Q} \\
P & =C_{2}-Q C_{1}
\end{aligned}
$$

with

$$
\mathcal{M}=\left[\begin{array}{c}
C_{2} \\
C_{1} \\
C_{1} A
\end{array}\right], \mathcal{M}_{\mathcal{N}}=\left[\begin{array}{c}
I_{q} \\
0_{m_{1} \times q} \\
0_{m_{1} \times q}
\end{array}\right], \mathcal{M}_{\mathcal{L}}=\left[\begin{array}{c}
0_{q \times m_{1}} \\
I_{m_{1}} \\
0_{m_{1} \times m_{1}}
\end{array}\right], \mathcal{M}_{\mathcal{Q}}=\left[\begin{array}{c}
0_{q \times m_{1}} \\
0_{m_{1} \times m_{1}} \\
I_{m_{1}}
\end{array}\right]
$$

where $K_{M} \in \mathbb{R}^{q \times\left(m+m_{1}\right)}$ is a gain matrix to be computed. Let $e=C_{2} x-\widehat{\nu}$ be the estimation error which dynamic's is driven by

$$
\dot{e}=N e+P(g(\nu, u, t)-g(\widehat{\nu}, u, t))
$$

The system (37) is a functional observer for the system (4) if conditions (2) and (38) are verified and if there exist matrices $X=X^{T}>0$ and $Z$ such that the following LMI is satisfied

$$
\left[\begin{array}{cc}
X N_{1}+N_{1}^{T} X-Z N_{2}-N_{2}^{T} Z^{T}+I_{q} & X\left(C_{2}-Q_{1} C_{1}\right)+Z Q_{2} C_{1} \\
\left(C_{2}-Q_{1} C_{1}\right)^{T} X+C_{1}^{T} Q_{2}^{T} Z^{T} & \frac{-1}{\lambda^{2}} I_{n}
\end{array}\right]<0
$$

with $K_{M}=X^{-1} Z$ and $N=N_{1}-K_{M} N_{2}$. Note first that the detectability of the pair $\left(N_{1}, N_{2}\right)$ is equivalent to condition (38b). In addition the LMI (41) is equivalent to the minimization of the $\mathcal{H}_{\infty}$ norm of the transfert function between $r=g(\nu, u, t)-g(\widehat{\nu}, u, t)$ and $e$ and can be expressed as $\left\|\left(s I_{q}-N\right)^{-1} P\right\|_{\infty}<\frac{1}{\lambda}$ (see $[6])$.

\section{Computation of the matrix $D$}

Following the previous development, we propose an algorithm to compute the matrices $D$ and $C_{1}$. Since the matrices $D$ and $\bar{D}$ are composed of $e_{i}$ (see notations in [1]), the problem consists in choosing the minimal number of rows $c_{i}$ of $C$ such that the rank condition (8) is satisfied. The index $i$ of the selected row $c_{i}$ corresponds to the column vector $e_{i}$ composing the matrix $\bar{D}$. Let $\Omega$ be the set of unstable eigenvalues of $A$.

Step 1 for $i=1: m$,

$C_{t}=c_{i}$

do TEST

end for $i$. 
Step 2 for $i=1: m-1$,

for $j=i+1: m$,

$C_{t}=\left[\begin{array}{c}c_{i} \\ c_{j}\end{array}\right]$

do TEST

end for $j$,

end for $i$,

Step 3 for $i=1: m-2$,

for $j=i+1: m-1$,

for $k=j+1: m$,

$C_{t}=\left[\begin{array}{c}c_{i} \\ c_{j} \\ c_{k}\end{array}\right]$

do TEST

end for $k$,

end for $j$,

end for $i$,

:

Step $\mathbf{m} C_{t}=C$, no sensor gain fault can be estimated.

STOP.

Step $\mathbf{m}+\mathbf{1} C_{1}=C_{t}$;

BREAK.

where

TEST : if $\operatorname{rank}\left[\begin{array}{c}\mu I_{n}-A \\ C_{t}\end{array}\right]=n, \quad \forall \mu \in \Omega$,

then goto step $m+1$.

Let us note that if there are $q$ conditionally identifiable sensors, then the maximum number $\bar{q}$ of combinations of rows $c_{i}$ to be tested is given by the following formula

$$
\bar{q}=\sum_{r=1}^{m-q} \mathrm{C}_{r}^{m}=\sum_{r=1}^{m-q} \frac{m !}{(m-r) ! r !}
$$

For the case treated in the example in [1], $m=4$ and $q=3$ then $\bar{q}=\frac{4 !}{3 !}=4$.

\section{Conclusion}

Through this discussion paper, we provide simple ideas to improve, in a way, the interesting work presented in [1].

\section{References}

[1] Y. Wang and D. Zhou, "Sensor gain fault diagnosis for a class of nonlinear systems," European J. Contr., vol. 12, pp. 523-535, 2006.

[2] M. Boutayeb, M. Darouach, and H. Rafaralahy, "Generalized state-space observers for chaotic synchronization and secure communication," IEEE Trans. Circ. Syst. I : Fund. Theory 83 Appli., vol. 49, pp. 345-349, 2002. 
[3] L. Dai, Singular Control Systems, vol. 118 of Lecture Notes in Control and Information Sciences. New York: Springer-Verlag, 1989.

[4] M. Darouach and M. Boutayeb, "Design of observers for descriptor systems," IEEE Trans. Aut. Contr., vol. 40, pp. 1323-1327, 1995.

[5] P. Lancaster and M. Tismenetsky, The Theory of Matrices. Orlando, USA: Academic Press, 2nd ed., 1985.

[6] R. Rajamani, "Observer for Lipschitz nonlinear systems," IEEE Trans. Aut. Contr., vol. 43, pp. 397401, 1998.

[7] F. Zhu and Z. Han, "A note on observers for Lipschitz nonlinear systems," IEEE Trans. Aut. Contr., vol. 47, pp. 1751-1754, 2002.

[8] M. Darouach, "Existence and design of functional observers for linear systems," IEEE Trans. Aut. Contr., vol. 45, pp. 940-943, 2000.

[9] H. Souley Ali, M. Darouach, and M. Zasadzinski, "Approche LMI pour la synthèse des filtres $\mathcal{H}_{\infty}$ non biaisés," in Proc. Conf. Int. Franc. Aut., (Bordeaux, France), 2006.

[10] M. Zasadzinski, H. Souley Ali, and M. Darouach, "Robust reduced order unbiased filtering for uncertain systems," Int. J. Contr., vol. 79, pp. 93-106, 2006. 